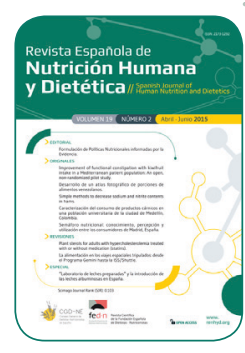

ORIGINAL

\title{
Semáforo nutricional: conocimiento, percepción y utilización entre los consumidores de Madrid, España
}

\author{
Karimen Andrea León-Flández ${ }^{a, *}$, Leandro Prieto-Castillo ${ }^{b}$, Miguel Angel Royo-Bordonada \\ a Hospital Clínico San Carlos, Madrid, España \\ b Escuela Nacional de Sanidad-Instituto de Salud Carlos III, Madrid, España. \\ *karimenleonf@gmail.com
}

Recibido el 10 de febrero de 2015; aceptado el 8 de mayo de 2015.

\section{PALABRAS CLAVE \\ Semáforo nutricional; \\ Etiquetado nutricional;}

Sistema de etiquetado frontal.
Semáforo nutricional: conocimiento, percepción y utilización entre los consumidores de Madrid, España

\section{RESUMEN}

Introducción: Describir el grado de conocimiento, comprensión, percepción y utilización del semáforo nutricional entre los consumidores madrileños.

Material y Métodos: Estudio transversal realizado en dos fases en el año 2012. Se reclutaron consumidores madrileños de forma aleatoria en zonas de espera de 7 supermercados. Mediante un cuestionario, se recogió información referente al conocimiento, percepción y utilización del semáforo nutricional. Se procedió al cálculo de porcentajes de las variables de interés y al análisis estratificado por variables sociodemográficas, aplicando el test de la Ji Cuadrado.

Resultados: La tasa de respuesta en la primera y segunda fase fue de $80,6 \%$ y $97,8 \%$ respectivamente. El $41,4 \%$ de los consumidores madrileños conocían el semáforo nutricional (fase 1 ) y el $18,6 \%$ comprendían su significado (fase 2 ). Entre los que lo conocían, al $61,5 \%$ les parecía muy útil ( $80 \%$ en mayores de 65 años y $90 \%$ en aquellos con estudios primarios; $p>0,05)$ y el $31,4 \%$ lo usaban habitualmente para comprar ( $70 \%$ en aquellos con estudios primarios; $p=0,04)$. Este porcentaje también fue superior entre los que lo comprendían $(66,6 \% ; p<0,01)$.

Conclusiones: El conocimiento y comprensión del semáforo nutricional fueron escasos. Los que lo conocen, lo consideran un instrumento muy útil para aportar información, y los que comprenden su significado, lo usan habitualmente al momento de la compra, sobre todo mayores de 65 años y con bajo nivel de estudios. La implantación generalizada de este sistema en España podría contribuir a mejorar hábitos y patrones alimentarios. 
Traffic light - nutrition labelling: knowledge, perception and use in consumers of Madrid, Spain

\section{KEYWORDS}

Traffic light labelling;

Nutritional labelling; Front of pack labelling.

\section{ABSTRACT}

Introduction: Describe knowledge, comprehension level, perception and use of traffic light labelling in consumers of Madrid.

Material and Methods: A two phases, cross-sectional study design, carried out in Madrid, in 2012. Our sample was recruited randomly, and interviewed using a questionnaire designed for this purpose. Consumers of 7 stores of main supermarket chains in Madrid were questioned. Information about knowledge, comprehension, perception and use of traffic light labelling (TLL) was collected. Analyses examined the frequency of the variables of interest. Differences were tested using the $\chi^{2}$ test.

Results: The response rate was $80.6 \%$ (first phase) and $97.8 \%$ (second phase). Consumers that knew the TLL and understood its color-coding system were $41.4 \%$ and $18.6 \%$ respectively. From the participants that knew the TLL system, $61.5 \%$ thought it was very useful $(80 \%$ among those $>65$ years and $90 \%$ among those with primary studies; $p>0.05$ ). Just $31.4 \%$ of consumers habitually used TLL to buy their products ( $70 \%$ among those with primary studies; $p=0.04)$. This percentage was higher in consumers that understood the color-coding system (second phase) (66.6\%; $p<0.01)$.

Conclusions: The level of TLL's knowledge and comprehension was low. Consumers that know TLL, consider it a useful nutrition instrument. Those who understand, use it habitually to buy their products, especially consumers older than 65 years old and with primary studies. A widespread introduction of this system in Spain might contribute to improve food and eating habits, especially elderly people and less educated.

\section{CITA}

León-Flández KA, Prieto-Castillo L, Royo-Bordonada MA. Semáforo nutricional: conocimiento, percepción y utilización entre los consumidores de Madrid, España. Rev Esp Nutr Hum Diet. 2015; 19 (2): 97 - 104. DOI: 10.14306/renhyd.19.2.150

\section{INTRODUCCIÓN}

El plan de acción global de la Organización Mundial de la Salud (OMS) para el control y prevención de enfermedades crónicas no transmisibles recomienda, entre otras medidas como promover una dieta saludable, la implementación del etiquetado nutricional en los alimentos ${ }^{1}$. Los escasos conocimientos nutricionales de la población, la falta de tiempo en el momento de la compra y la complejidad en la forma de presentar la información nutricional dificultan con frecuencia la elección de alimentos saludables².

En respuesta a la necesidad de disponer de sistemas de información simples, de fácil comprensión y utilización, la
Agencia de Normas Alimentarias del Reino Unido desarrolló el semáforo nutricional (SN), un sistema de etiquetado frontal que indica, mediante un código de colores (rojo o naranja, amarillo y verde), si la cantidad de calorías y determinados nutrientes (grasas saturadas, azúcar y sal) de un producto alimentario son excesivas, considerándose a éste más o menos saludable ${ }^{2-4}$.

Diversos estudios, incluido uno recientemente llevado a cabo con adolescentes españoles ${ }^{5}$, sugieren que el SN es el tipo de etiquetado más útil para identificar alimentos saludables, ya que permite hacer un juicio rápido de la calidad nutricional de los alimentos, especialmente a las personas que no disponen del tiempo suficiente o de los conocimientos nutricionales necesarios para ello ${ }^{6-9}$. 
En 2011, la Unión Europea adoptó el etiquetado nutricional obligatorio de los alimentos envasados ${ }^{10}$. Sin embargo, la fuerte oposición de la industria alimentaria impidió la implantación del semáforo nutricional, largo tiempo demandada por asociaciones de consumidores y organizaciones del mundo de la salud ${ }^{11}$. A diferencia del Reino Unido, donde el gobierno está promoviendo la implantación del SN12, en España no existe actualmente ninguna iniciativa pública en este sentido, siendo la implantación de este sistema de etiquetado muy escasa.

Hasta donde llega el conocimiento de los autores del presente artículo, a nivel nacional no se han realizado muchos estudios que evalúen este tipo de etiquetado nutricional. Recientemente se publicó un estudio realizado en adolescentes de 14 a 16 años en un colegio, el cual mostró que cuando éstos utilizaron el SN seleccionaron alimentos más saludables con respecto a otro tipo de etiquetado nutricional ${ }^{5}$. En 2007, la cadena de supermercados Eroski, a iniciativa de los consumidores implantó el SN en sus productos de marca propia $^{13}$. Posteriormente, publicó un artículo de carácter divulgativo con una limitada descripción metodológica, que sugiere un alto grado de conocimiento y uso del SN entre sus consumidores. Aunque se trata de un estudio a nivel nacional, la no inclusión de consumidores de otras cadenas distribuidoras de alimentos limita su representatividad y la posible generalización de sus conclusiones ${ }^{14}$.

Este estudio tiene por objeto describir el grado de conocimiento, comprensión, percepción y utilización del semáforo nutricional en una muestra de consumidores de las principales cadenas de supermercados de alimentación de Madrid.

\section{MATERIAL Y MÉTODOS}

\section{Diseño del estudio}

Estudio transversal realizado en dos fases. La primera, entre marzo y mayo de 2012, con una muestra de consumidores de 5 supermercados de las principales cadenas de alimentación de Madrid, situados en los barrios del Pilar (Alcampo), Almenara (Día), Moncloa (Eroski) y Mirasierra (Gama y Maxcoop). Se analizó el conocimiento, percepción y utilización del SN. La segunda, en noviembre y diciembre de 2012, con una muestra de consumidores de 2 supermercados Eroski, situados en los barrios de Chamberí y Salamanca. En esta fase se analizó el grado de comprensión del código de colores del $\mathrm{SN}$, percepción y utilización del mismo. En esta fase, con el objetivo de obtener una muestra de consumidores similar en tamaño al número de consumidores de supermercados Eroski de la primera fase ${ }^{15}$, se calculó el tamaño muestral necesario para un porcentaje estimado de comprensión del semáforo nutricional del 40\%, con un error de precisión del $7 \%$ y un intervalo de confianza del 90\% $(\mathrm{N}=132)$.

\section{Recogida de información}

Se diseñó un cuestionario ad hoc basado en estudios ${ }^{16-18}$ similares a nivel internacional, mediante el que se recogió información sociodemográfica y la concerniente al interés, comprensión y utilización del etiquetado nutricional, con un apartado dedicado al SN. Para evaluar el grado de comprensión del cuestionario y la factibilidad de su aplicación en el punto de venta, se realizó un estudio piloto sobre una muestra de 20 personas en dos de los supermercados seleccionados (Día y Eroski), comprobando que la encuesta podía llevarse a cabo en un tiempo medio estimado de 5 a 7 minutos en las zonas de espera, como línea de caja, pescadería, charcutería y similares.

Los 5 encuestadores que participaron en el estudio, previamente instruidos, cumplimentaron los cuestionarios mediante entrevistas personales a los consumidores elegidos al azar dentro de los supermercados, variando el horario y el día de la semana. Antes de realizar la entrevista, se informaba a los consumidores del motivo de la encuesta y se les invitaba a participar. En caso de negativa, se invitaba a participar al siguiente consumidor de la fila. El encuestador leía cada pregunta, junto con sus opciones de respuesta, de forma que el entrevistado debía elegir una de entre esas categorías cerradas.

En la primera fase, en el apartado relativo al SN, antes de formular la pregunta sobre el conocimiento del mismo, se le mostraba al entrevistado un etiquetado de un producto de marca propia de Eroski con un semáforo nutricional múltiple. A los que afirmaron conocerlo, se les preguntó acerca de la percepción de su utilidad y el uso del mismo en el momento de la compra. Ante el posible riesgo de que se pudiera haber introducido un sesgo (por inducción a una respuesta positiva) al mostrar la etiqueta antes de las preguntas, en la segunda fase, la pregunta relacionada con el conocimiento del SN se realizó antes de mostrar la etiqueta. A los que afirmaron conocerlo, se les preguntó acerca del significado del código de colores (comprensión objetiva) y, en caso de obtener una respuesta correcta, a continuación se completaban el resto de preguntas, relativas a la percepción de la utilidad y uso del mismo en el momento de la compra.

\section{Análisis de los datos}

Se analizó el perfil de los consumidores y se procedió al análisis descriptivo de los datos, mediante la distribución de frecuencias del grado de conocimiento o comprensión de $\mathrm{SN}$, según la fase del estudio. Entre los consumidores que conocían o comprendían el SN, según la fase del estudio, se estimaron los porcentajes de los que tenían una opinión 
favorable de la utilidad del mismo y los que lo utilizaban en el momento de la compra. A continuación se realizó un análisis estratificado por las variables sociodemográficas, aplicando el test de la Ji Cuadrado para la comparación de proporciones. Cuando las frecuencias esperadas en alguna de las categorías fueron demasiado bajas, se utilizó el test exacto de Fisher. Todos los análisis se realizaron con el paquete estadístico PASW Statistic 20 (SPSS Inc., Chicago, IL, USA).

\section{$\longrightarrow$ RESULTADOS}

De los 371 consumidores invitados a participar en la primera fase, aceptaron un $80,6 \%$. De estos, el 59,5\% fueron mujeres. La edad media de los participantes fue de 45,7 años (D.E.:15.2). En la segunda fase del estudio, se invitaron a participar a 132 personas, de los cuales aceptaron un 97,8\%, con una edad media de 47,9 años (D.E.:14.4).

En ambas fases del estudio, la mayoría de participantes fueron mujeres, de nacionalidad española, casados o con pareja, sin hijos menores de 18 años a su cargo, trabajadores en activo y con estudios universitarios. Los participantes en la primera fase fueron más jóvenes en promedio $(p=0,03)$ y con un nivel de estudios más bajo $(p=0,03)$, comparados con los de la segunda fase (Tabla 1).

El porcentaje de consumidores que conocían el SN (primera fase) fue del $41,4 \%$. Este porcentaje fue ligeramente más bajo entre los consumidores de Eroski $(37,5 \%)$ y más alto en hombres $(44,6 \%)$, extranjeros $(53,5 \%)$, casados o conviviendo en pareja (45,2\%), con hijos menores de 18 años a su cargo $(48,4 \%)$, con trabajo $(43,5 \%)$ y con estudios universitarios (42,7\%); aunque las diferencias no fueron estadísticamente significativas en ningún caso (Tabla 2). El porcentaje de consumidores que comprendía el SN (segunda fase) fue del $18,6 \%$. Así como en la primera fase, el porcentaje de comprensión fue más alto en hombres $(22,6 \%)$, con hijos menores de 18 años a su cargo (23,9\%), con trabajo (22,6\%) y estudios universitarios (19,2\%). Se mostró un porcentaje mayor de comprensión del SN en españoles que en extranjeros ( $20 \%$ vs. $12,5 \%$ respectivamente) y en solteros que en casados o conviviendo en pareja (21,1\% vs. 19,4\% respectivamente); aunque las diferencias no fueron estadísticamente significativas en ningún caso (Tabla 2).

En la primera fase, al $61,5 \%$ de los consumidores que conocían el SN, les parecía un sistema de etiquetado muy útil para transmitir la información nutricional. Esta cifra fue superior en mayores de 65 años (80\%) y con estudios primarios $(90 \%)$, aunque las diferencias no fueron estadísticamente significativas (datos no mostrados en la tabla). El 31,4\% de los consumidores de esta fase declararon que siempre o casi siempre utilizaban el SN para comprar. Esta cifra fue superior en mayores (40\%) y con estudios primarios $(70 \%)$, siendo el nivel de estudios estadísticamente significativo ( $p=0,04 ;$ datos no mostrados en la tabla).

En la segunda fase, al $75 \%$ de los consumidores que comprendían el SN, les parecía un sistema de etiquetado muy útil y a un $25 \%$ algo útil para transmitir información nutricional. El 66,6 \% de los consumidores que comprendían el SN lo utilizaban para comprar siempre o casi siempre y el 16,7\% nunca (Tabla 3).

Los consumidores que comprendían el SN, lo consideraron con mayor frecuencia muy útil ( $75 \%$ vs. $61,5 \%)$ y lo utilizaban más (66,6\% vs. $31,4 \%$ siempre o casi siempre) con respecto a los que declaraban conocerlo, siendo esta última diferencia estadísticamente significativa $(p<0.01)$ (Tabla 3).

\section{DIscusión Y CONCLUSIONES}

Por encima del $40 \%$ de los consumidores madrileños declararon conocer el semáforo nutricional; mientras que el grado de comprensión objetiva (fase 2) del mismo fue muy inferior, situándose por debajo del 20\%. Entre los consumidores que declararon conocer el SN, los mayores de 65 años, con nivel de estudios primarios y los que comprendían el significado del código de colores, fueron los que con mayor frecuencia consideraron a este sistema de etiquetado como muy útil para transmitir información nutricional de los productos alimentarios y lo utilizaban de forma más habitual en el momento de la compra.

El nivel de conocimiento del SN entre los consumidores del presente estudio (41,4\%) fue muy inferior al del estudio Eroski (56\%), realizado en $2013^{14}$. Aunque el estudio Eroski es de ámbito nacional, es posible que esta diferencia se deba a que la muestra se reclutó un año más tarde que la del presente estudio. Sin embargo, entre los que declararon conocerlo, la mayoría lo consideraban un sistema de información útil y lo usaban en el momento de la compra, con cifras similares a las del estudio Eroski. El conocimiento y comprensión del SN en el presente estudio fueron muy bajos en comparación con los de un estudio realizado en el Reino Unido en 2009, donde el 87,5\% de los encuestados eran capaces de identificar productos saludables gracias al etiquetado nutricional ${ }^{19}$, diferencias posiblemente debidas a la mayor implantación de este sistema de etiquetado en ese país ${ }^{12}$. Entre los consumidores que declararon conocer el SN, el uso del mismo fue mucho menor que entre los que comprendían el significado de su código de colores, donde se obtuvieron porcentajes más acordes con los de otros estudios internacionales ${ }^{20-21}$, pues el nivel declarado de conocimiento del semáforo no se corresponde con el grado de compresión del significado de su código de colores. 
Tabla 1. Características sociodemográficas de la muestra de consumidores madrileños (fases 1 y 2 del estudio)

\begin{tabular}{|c|c|c|c|}
\hline & $\begin{array}{c}\text { FASE } 1 \\
\mathrm{n}(\%)\end{array}$ & $\begin{array}{c}\text { FASE } 2 \\
\mathrm{n}(\%)\end{array}$ & p valorb \\
\hline \multicolumn{4}{|l|}{ Supermercado } \\
\hline Eroski & $89(29,8)$ & $130(100,0)$ & \multirow[t]{2}{*}{$<0,001$} \\
\hline Otros ${ }^{c}$ & $210(70,2)$ & $0(0,0)$ & \\
\hline \multicolumn{4}{|l|}{ Sexo } \\
\hline Hombre & $121(40,5)$ & $53(40,8)$ & \multirow[t]{2}{*}{0,953} \\
\hline Mujer & $178(59,5)$ & $77(59,2)$ & \\
\hline \multicolumn{4}{|l|}{ Nacionalidad } \\
\hline Española & $252(84,8)$ & $106(81,5)$ & \multirow[t]{2}{*}{0,392} \\
\hline Extranjera & $45(15,2)$ & $24(18,5)$ & \\
\hline \multicolumn{4}{|l|}{ Edad (años) } \\
\hline $18-35$ & $95(31,8)$ & $26(20,0)$ & \multirow[t]{4}{*}{0,025} \\
\hline $36-50$ & $85(28,4)$ & $54(41,5)$ & \\
\hline $51-64$ & $86(28,8)$ & $35(26,9)$ & \\
\hline$>65$ & $33(11,0)$ & $15(11,5)$ & \\
\hline \multicolumn{4}{|l|}{ Estado Civil } \\
\hline Soltero & $82(27,5)$ & $39(30,5)$ & \multirow[t]{3}{*}{0,788} \\
\hline Casado / Convive en pareja & $178(59,7)$ & $72(56,3)$ & \\
\hline Separado / Divorciado / Viudo & $38(12,8)$ & $17(13,3)$ & \\
\hline \multicolumn{4}{|l|}{ Personas en domicilio } \\
\hline Vive solo & $25(8,5)$ & $18(13,8)$ & \multirow[t]{3}{*}{0,321} \\
\hline Con $1-2$ personas & $165(55,9)$ & $66(50,8)$ & \\
\hline Con $>2$ personas & $105(35,6)$ & $46(35,4)$ & \\
\hline \multicolumn{4}{|l|}{ Hijos < 18 años } \\
\hline No & $207(69,2)$ & $83(64,3)$ & \multirow[t]{2}{*}{0,237} \\
\hline Sí & $92(30,8)$ & $46(35,7)$ & \\
\hline \multicolumn{4}{|l|}{ Actividad económica } \\
\hline Trabaja & $192(64,4)$ & $84(65,1)$ & \multirow[t]{4}{*}{0,390} \\
\hline En desempleo & $56(18,8)$ & $20(15,5)$ & \\
\hline Estudiante & $18(6,0)$ & $5(3,9)$ & \\
\hline Jubilado & $32(10,7)$ & $20(15,5)$ & \\
\hline \multicolumn{4}{|l|}{ Nivel de estudios } \\
\hline Primarios & $29(9,7)$ & $3(2,3)$ & \multirow[t]{3}{*}{0,028} \\
\hline Secundarios & $98(32,8)$ & $47(36,4)$ & \\
\hline Universitarios & $172(57,5)$ & $79(61,2)$ & \\
\hline TOTAL & $299(100,0)$ & $130(100,0)$ & \\
\hline
\end{tabular}

a: En la fase 1 se evaluó el grado de conocimiento y en la fase 2 el grado de comprensión del SN. En ambas fases, a aquellos que conocían/comprendían el SN se les preguntó por su percepción de la utilidad para transmitir información nutricional y por el uso del mismo al momento de realizar la compra.

b: Test de la $\chi^{2}$ para comparación de proporciones. Cuando las frecuencias esperadas fueron demasiado bajas, se utilizó el test exacto de Fisher.

c: Otros: Alcampo, DIA, Gama, Maxcoop. 
Rev Esp Nutr Hum Diet. 2015; 19(2): 97 - 104

Semáforo nutricional: conocimiento, percepción y utilización entre los consumidores de Madrid, España

Tabla 2. Grado de conocimiento (Fase 1) y comprensión (Fase 2) del SN, en función de características sociodemográficas.

\begin{tabular}{|c|c|c|c|c|c|c|}
\hline & \multicolumn{3}{|c|}{ FASE 1} & \multicolumn{3}{|c|}{ FASE 2} \\
\hline & $\begin{array}{c}\text { Conoce } \\
\text { n (\%) }\end{array}$ & $\begin{array}{c}\text { TOTAL } \\
\text { N }\end{array}$ & p valora & $\begin{array}{c}\text { Comprende } \\
n(\%)\end{array}$ & $\begin{array}{c}\text { TOTAL } \\
\text { N }\end{array}$ & p valor ${ }^{a}$ \\
\hline \multicolumn{7}{|l|}{ Supermercado } \\
\hline Eroski & $33(37,5)$ & 88 & \multirow[t]{2}{*}{0.374} & $24(18,6)$ & 129 & \\
\hline Otros $^{b}$ & $90(43,1)$ & 209 & & $0(0,0)$ & 0 & \\
\hline \multicolumn{7}{|l|}{ Sexo } \\
\hline Hombre & $54(44,6)$ & 121 & \multirow[t]{2}{*}{0.351} & $12(22,6)$ & 53 & \multirow[t]{2}{*}{0,325} \\
\hline Mujer & $69(39,2)$ & 176 & & $12(15,8)$ & 76 & \\
\hline \multicolumn{7}{|l|}{ Nacionalidad } \\
\hline Española & $100(39,7)$ & 252 & \multirow[t]{2}{*}{0.090} & $21(20,0)$ & 105 & \multirow[t]{2}{*}{0,564} \\
\hline Extranjera & $23(53,5)$ & 43 & & $3(12,5)$ & 24 & \\
\hline \multicolumn{7}{|l|}{ Edad (años) } \\
\hline $18-35$ & $39(41,5)$ & 94 & \multirow[t]{4}{*}{0.567} & $5(20,0)$ & 25 & \multirow[t]{4}{*}{0,709} \\
\hline $36-50$ & $36(42,9)$ & 84 & & $11(20,4)$ & 54 & \\
\hline $51-64$ & $38(44,2)$ & 86 & & $7(20,0)$ & 35 & \\
\hline$>65$ & $10(30,3)$ & 33 & & $1(6,7)$ & 15 & \\
\hline \multicolumn{7}{|l|}{ Estado Civil } \\
\hline Soltero & $28(34,6)$ & 81 & \multirow[t]{3}{*}{0.264} & $8(21,1)$ & 38 & \multirow[t]{3}{*}{0,439} \\
\hline Casado / Convive en pareja & $80(45,2)$ & 177 & & $14(19,4)$ & 72 & \\
\hline Separado / Divorciado / Viudo & $15(39,5)$ & 38 & & $1(5,9)$ & 17 & \\
\hline \multicolumn{7}{|l|}{ Hijos $<18$ años } \\
\hline No & $79(38,3)$ & 206 & \multirow[t]{3}{*}{0.107} & $13(15,9)$ & 82 & \multirow[t]{3}{*}{0,262} \\
\hline Sí & $44(48,4)$ & 91 & & $11(23,9)$ & 46 & \\
\hline \multicolumn{5}{|l|}{ Personas en domicilio } & & \\
\hline Vive solo & $7(28,0)$ & 25 & \multirow[t]{3}{*}{0.111} & $2(11,1)$ & 18 & \multirow[t]{3}{*}{0,447} \\
\hline Con $1-2$ personas & $63(38,4)$ & 164 & & $15(23,1)$ & 65 & \\
\hline Con $>2$ personas & $51(49,0)$ & 104 & & $7(15,2)$ & 46 & \\
\hline \multicolumn{7}{|l|}{ Actividad económica } \\
\hline Trabaja & $83(43,5)$ & 191 & \multirow[t]{4}{*}{0.500} & $19(22,6)$ & 84 & \multirow[t]{4}{*}{0,455} \\
\hline En desempleo & $23(41,8)$ & 55 & & $2(10,0)$ & 20 & \\
\hline Estudiante & $5(27,8)$ & 18 & & $1(20,0)$ & 5 & \\
\hline Jubilado & $11(34.4)$ & 32 & & $2(10,0)$ & 20 & \\
\hline \multicolumn{7}{|l|}{ Nivel de estudios } \\
\hline Primarios & $10(34,5)$ & 29 & \multirow[t]{3}{*}{0.708} & $0(0,0)$ & 3 & 0,899 \\
\hline Secundarios & $40(41,2)$ & 97 & & $8(17,0)$ & 47 & \\
\hline Universitarios & $73(42,7)$ & 171 & & $15(19,2)$ & 78 & \\
\hline TOTAL & $123(41,4)$ & 297 & & $24(18,6)$ & 129 & \\
\hline
\end{tabular}

a: Test de la $\chi^{2}$ para comparación de proporciones. Cuando las frecuencias esperadas fueron demasiado bajas, se utilizó el test exacto de Fisher.

b: Otros: Alcampo, DIA, Gama, Maxcoop 
Tabla 3. Utilidad del SN para transmitir información nutricional y uso del mismo en el momento de la compra, en función del conocimiento (Fase 1) y comprensión (Fase 2).

\begin{tabular}{l|c|c|c} 
& FASE 1 & FASE 2 & \\
\hline & $\begin{array}{c}\text { Conoce } \\
\mathrm{n}(\%)\end{array}$ & $\begin{array}{c}\text { Comprende } \\
\mathrm{n}(\%)\end{array}$ & $\mathrm{p}$ valor \\
\hline Utilidad para informar & & & \\
\hline Mucho & $75(61,5)$ & $18(75,0)$ & 0,376 \\
Algo & $39(32,0)$ & $6(25,0)$ & \\
Poco / Nada & $8(6,5)$ & $0(0,0)$ & \\
\hline Uso para comprar & $38(31,4)$ & $16(66,6)$ & $<0,001$ \\
\hline Siempre / La mayoría de veces & $49(40,5)$ & $4(16,7)$ & \\
Ocasionalmente & $34(28,1)$ & $4(16,7)$ & \\
Nunca & $\mathbf{1 2 1 ( 1 0 0 , 0 )}$ & $\mathbf{2 4 ( 1 0 0 , 0 )}$ & \\
TOTAL & & & \\
\hline
\end{tabular}

a: Test de la $\chi^{2}$ para comparación de proporciones. Cuando las frecuencias esperadas fueron demasiado bajas, se utilizó el test exacto de Fisher.

Los resultados mostrados en este estudio coinciden con los de otras investigaciones en que las personas de edad avanzada y con niveles de estudios inferiores son las más interesadas en el SN y las que hacen un mayor uso del mismo en el momento de la compra ${ }^{20-22}$. Se ha observado que la compresión del etiquetado tiene sobre todo que ver con el nivel de conocimientos nutricionales, mientras que el uso del mismo tiene más relación con el interés por la asociación entre alimentación y salud, viéndose no obstante influida la decisión de comprar alimentos más saludables por el nivel de estudios y el nivel socioeconómico: a niveles más bajos se opta por alimentos ricos en azúcares y grasas saturadas, ya que estos son más baratos, accesibles y de preparación más rápida y fácil19. Las mujeres de edad avanzada se preocupan más por su salud y por la relación de la alimentación con las enfermedades crónicas ${ }^{21,23}$, pero poseen menos conocimientos nutricionales ${ }^{24}$. Esto explicaría que tengan una mejor percepción y hagan un mayor uso de un sistema de información sencillo, como el semáforo, cuya comprensión apenas requiere conocimientos nutricionales. EI SN en estos casos resulta especialmente útil, ya que es de fácil y rápida comprensión ${ }^{25}$, permitiendo optar por alimentos más saludables en el momento de la compra $a^{5,7,9,26}$.

La principal limitación del presente estudio fue la reducida representatividad de la muestra, pues el nivel de estudios fue muy superior a la media de la población madrileña ${ }^{27}$ debido a la inclusión de barrios de nivel socioeconómico medioalto y a la exclusión de pequeños comercios de barrio. Además, puede que los consumidores que aceptaron participar estuvieran más sensibilizados con el tema. Por todo ello, es posible que se haya producido una sobreestimación en los porcentajes de nuestras variables dependientes. Sin embargo, se buscó la mayor representatividad posible incluyendo las principales cadenas de supermercados que operan en Madrid y procediendo, dentro de cada establecimiento, a la selección aleatoria de los participantes. La elevada tasa de respuesta y el hecho de que las asociaciones encontradas sean consistentes con las de otros estudios, sugiere que de haberse producido algún tipo de sesgo de no respuesta, éste sería no diferencial.

En el presente estudio, el nivel de conocimiento y comprensión del SN fue relativamente bajo entre los consumidores madrileños, probablemente debido a la escasa implantación de este sistema de etiquetado en España. No obstante, aquellos que lo conocen lo consideran un instrumento muy útil para aportar información, y los que comprenden el significado del código de colores, hacen uso del mismo de forma habitual en el momento de la compra, sobre todo personas mayores y con bajo nivel de estudios. Teniendo en cuenta que el semáforo nutricional es el tipo de etiquetado más útil para identificar alimentos saludables y que los consumidores escogen hasta cinco veces más frecuentemente alimentos saludables con el semáforo nutricional que con el formato basado en porcentajes de ingesta diaria recomendada ${ }^{5,28}$, la implantación generalizada de este sistema en España podría contribuir a fomentar hábitos alimentarios más saludables, particularmente entre las personas mayores y/o con menor nivel de estudios, que son las más afectados por enfermedades relacionadas con una alimentación no saludable. 


\section{$\longrightarrow$ CONFLICTO DE INTERESES}

Los autores expresan que no hay conflictos de intereses al redactar el manuscrito.

\section{BIBLIOGRAFÍA}

1. World Health Organization (WHO). Follow-up to the Political Declaration of the High-level Meeting of the General Assembly on the Prevention and Control of Non communicable Diseases. WHA 66.10; 2013. Disponible en: http://apps.who.int/gb/ ebwha/pdf files/WHA66/A66 R10-en.pdf

2. Feunekes $\bar{G} I J$, Gortemaker $\bar{A} A$, Willems $A A$, Lion $R$, van den Kommer $M$. Front-of-pack nutrition labelling: Testing effectiveness of different nutrition labelling formats frontofpack in four European countries. Appetite. 2008; 50(1): 5770.

3. Food Standard Agency. Front of pack. Traffic Light signposting labelling. Technical Guidance. noviembre de 2007;(2). Disponible en: http://www.food.gov.uk/multimedia/pdfs/ frontofpackguidance2.pdf

4. Méjean C, Macouillard P, Péneau S, Hercberg S, Castetbon K. Perception of front-of-pack labels according to social characteristics, nutritional knowledge and food purchasing habits. Public Health Nutr. 2013; 16(3): 392-402.

5. Babio N, Vicent P, López L, Benito A, Basulto J, Salas-Salvadó ]. Adolescents' ability to select healthy food using two different front-of-pack food labels: a cross-over study. Public Health Nutr. 2014; 17(6): 1403-9.

6. Borgmeier I, Westenhoefer J. Impact of different food label formats on healthiness evaluation and food choice of consumers: a randomized-controlled study. BMC Public Health. 2009; 9(1): 184.

7. Kelly B, Hughes C, Chapman K, Louie JC-Y, Dixon H, Crawford J, et al. Consumer testing of the acceptability and effectiveness of front-of-pack food labelling systems for the Australian grocery market. Health Promot Int. 2009; 24(2): 120-9.

8. Thorndike AN, Sonnenberg L, Riis J, Barraclough S, Levy DE. A 2-Phase Labeling and Choice Architecture Intervention to Improve Healthy Food and Beverage Choices. Am J Public Health. 2012; 102(3): 527-33.

9. Magnusson RS. Obesity prevention and personal responsibility: the case of front-of-pack food labelling in Australia. BMC Public Health. 2010; 10(1): 662.

10. Boletín Oficial del Estado. Reglamento (UE) No 1169/2011 del Parlamento Europeo y el Consejo de 25 de octubre de 2011. Disponible en: http://aesan.msssi.gob.es/AESAN/docs/docs/ notas_prensa/reglamento_jornada_consumidor.pdf

11. EurActiv | EU News \& policy debates, across languages. Food industry wins battle on "traffic light» labels. En prensa 2010. Disponible en: http://www.euractiv.com/food-industrywinsbattle-traffic-light-labels-news-495324

12. Department of Health (UK). Final design of consistent nutritional labelling system given green light. Junio 2013. Disponible en: https://www.gov.uk/government/news/ finaldesign- of-consistent-nutritional-labelling-system-givengreenlight

13. Navarro J. Los productos de marca blanca de Eroski llevarán un semáforo nutricional. En prensa 2007. Disponible en: http://www.casacochecurro.com/20070911372/vivienda/ los-productos-de-marca-blanca-de-eroski-llevarunsemronutricional.html

14. Cuevas R. Etiquetado nutricional: útil pero complejo. Eroski Consumer. En prensa 2013. Disponible en: http://revista. consumer.es/web/es/20131001/pdf/revista_entera.pdf

15. Prieto-Castillo L, Royo-Bordonada MA, Moya-Geromini A. Information search behaviour, understanding and use of nutrition labeling by residents of Madrid, Spain. Public Health. 2015; 129(3): 226-36.

16. Lynam AM, McKevitt A, Gibney MJ. Irish consumers' use and perception of nutrition and health claims. Public Health Nutr.2011; 14(12): 2213-9.

17. Chen X, Jahns L, Gittelsohn J, Wang Y. Who is missing the message? Targeting strategies to increase food label use among US adults. Public Health Nutr. 2012; 15(5): 760-72.

18. Souza SM, Lima KC, Miranda HF, Cavalcanti FI. [Consumer use of nutrition labels in the city of Natal, Brazil]. Rev Panam Salud Publica. 2011; 29(5): 337-43.

19. Grunert KG, Wills JM, Fernández-Celemín L. Nutrition knowledge, and use and understanding of nutrition information on food labels among consumers in the UK. Appetite. 2010; 55(2): 177-89.

20. Grunert KG, Wills JM. A review of European research onconsumer response to nutrition information on food labels. J Public Health. 2007; 15(5): 385-99.

21. Cowburn G, Stockley L. Consumer understanding and use of nutrition labelling: a systematic review. Public Health Nutr. 2005; 8(1): 21-8.

22. Besler HT, Buyuktuncer Z, Uyar MF. Consumer Understanding and Use of Food and Nutrition Labeling in Turkey. J Nutr Educ Behav. 2012; 44(6): 584-91.

23. Lewis JE, Arheart KL, LeBlanc WG, Fleming LE, Lee DJ, Davila EP, et al. Food label use and awareness of nutritional information and recommendations among persons with chronic disease. Am J Clin Nutr. 2009; 90(5): 1351-7.

24. Grunert KG, Fernández-Celemín L, Wills JM, Storcksdieck genannt Bonsmann S, Nureeva L. Use and understanding of nutrition information on food labels in six European countries. J Public Health. 2010; 18(3): 261-77.

25. Triggle N. Food labels system to be rolled out. BBC News Health. En prensa 2013. Disponible en: http://www.bbc.co.uk/ news/health-22959239

26. Gerrior SA. Nutrient profiling systems: are science and the consumer connected? Am J Clin Nutr. 2010; 91(4): 1116S-1117S.

27. Instituto de Salud Pública de Madrid. Encuesta de salud de la ciudad de Madrid (2004-2005); 2005. Disponible en: http:// www.madridsalud.es/publicaciones

28. Kelly B, Hughes C, Chapman K, Louie J, Dixon H, King L. Front-of-Pack Food Labelling: Traffic Light Labelling Gets the Green Light. Cancer Counsil 2008. Disponible en: http://www. cancercounsil.com.au/nutrition/foodlabellingreport 